\title{
Immune-checkpoint inhibitors and the importance of concomitant medications: focus on antibiotics
}

\author{
Chiara Casadei, Cristian Lolli, Alberto Farolfi \\ Department of Medical Oncology, Istituto Scientifico Romagnolo per lo Studio e la Cura dei Tumori (IRST) IRCCS, Italy \\ Correspondence to: Dr. Alberto Farolfi, MD. Department of Medical Oncology, Istituto Scientifico Romagnolo per lo Studio e la Cura dei Tumori \\ (IRST) IRCCS, Italy. Email: alberto.farolfi@irst.emr.it. \\ Provenance: This is an invited article commissioned by the Section Editor Dr. Xiao Li (Department of Urology, Jiangsu Cancer Hospital, Jiangsu \\ Institute of Cancer Research, Nanjing Medical University Affiliated Cancer Hospital, Nanjing, China). \\ Comment on: Tinsley N, Zhou C, Tan G, et al. Cumulative Antibiotic Use Significantly Decreases Efficacy of Checkpoint Inhibitors in Patients with \\ Advanced Cancer. Oncologist 2019. [Epub ahead of print].
}

Submitted Sep 09, 2019. Accepted for publication Sep 18, 2019.

doi: 10.21037/atm.2019.09.99

View this article at: http://dx.doi.org/10.21037/atm.2019.09.99

Harnessing the immune system to battle cancer, long a medical utopia, is becoming a reality. With the advent of immunotherapy, substantial progresses have been made in improving patients outcome, but only for a minority of them. Predicting responses to immunotherapy remains an open question, because not all patients benefit equally from treatment, and identification of predictive biomarkers for this class of agents is currently a challenge. In this context, tumor mutational burden (TMB), mismatch repair status (MMR), programmed cell death ligand 1 (PD-L1) expression, tumor-infiltrating lymphocytes (TILs), and inflammatory markers such as neutrophil-to-lymphocyte ratio (NLR) have been proposed but none of them is validated. Tumor microenvironment (TILs and PD-L1 expression) as well as mutational status or neoantigen load (TMB and MMR) are investigated extensively. Recently, patient-associated factors such as sex, age, body mass index (BMI) and gut microbiota demonstrated to profoundly influence immune responses.

The lowest common denominator of host-related factors seems inflammation. For example, the adipose tissue modulates the Th1/Th2 balance, decreases the activation of Treg through adiponectin, increases pro-inflammatory macrophages and increases inflammation, resulting in a negative impact on cancer prognosis (1). Nevertheless, immune checkpoint-inhibitor (ICI) may be more efficacy against such inflamed and immune-exhausted status. Thus, this could be the reason why patients with a $\mathrm{BMI} \geq 25$ seems to experience a better clinical outcome with anti-PD-1/PD-
L1 agents, compared to normal weight patients $(2,3)$.

Similarly, systemic inflammatory markers, such as NLR, are able to assess the balance between neutrophil-dependent pro-tumor inflammation and lymphocyte-associated antitumor immune response. Thus, inflammatory indexes such as NLR could serve as a prognostic factor and could be a helpful predictive tool, when validated in prospective trials $(4,5)$. Another promising inflammatory index, the systemic immune inflammation index (SII), based on neutrophil, lymphocyte and platelet counts has been associated with poor outcome, representing a useful prognostic indicator $(6,7)$. In fact, granulocyte-colony stimulating factor (G-CSF) and granulocyte-macrophage-colony stimulating factor (GM-CSF) induced by the tumor increase myeloid cells. Neutrophils and myeloid-derived suppressor cells (MDSC) can release several cytokines, such as arginase-1, which is an enzyme that can inhibit $\mathrm{T}$ cell responses and $\mathrm{T}$ cell proliferation. This could offer the immunological basis for the clinical observation that a high NLR is correlated with poor survival in several malignancies (3-5) and with reduced effectiveness of ICI therapy (8).

Furthermore, it has been recognized that gut microbiota is able to exert a powerful influence on response to immunotherapy (9). When dysregulated, the gut microbiota contributes to alter systemic immune responses, potentially favoring the development of chronic inflammatory disorders such as obesity, Crohn's disease and type II diabetes. Preclinical studies revealed that the anticancer activity of anti-CTLA-4, anti-PD-1/PD-L1 or the 
combination of both antibodies was lost in the presence of a low immunogenic gut microbiota, building on the hypothesis that its composition may determine resistance to ICIs. Indeed, intestinal microbiota from advanced melanoma, NSCLC and RCC patients that progressed to immunotherapy differed from those who responded (10). Moreover, gut microbiota seems to control immune-related adverse events after a preliminary study that reported promising data in patients with refractory immunotherapyassociated colitis. Fecal microbiota transplantation helped to recover from this adverse event, successfully reconstituting the gut microbiome and increasing the proportion of Tregs within the colonic mucosa (11).

Since immunotherapy indications continue to expand, medical community would face new challenges in patient management due to interactions with concomitant medications. Indeed, corticosteroids (excluding low doses for short periods) were prohibited in almost all the pivotal clinical trials with ICIs. Additionally, most studies do not report efficacy or safety data of immunotherapy in relation to the administration of drugs used routinely, such as antibiotics (ATBs) and proton pump inhibitors or their impact on gut microbiota.

ATBs represent frequent concurrent medications during cancer treatment and are undoubtedly drugs that may alter gut microbiota leading to dysbiosis and influencing immune responses. Preclinical studies demonstrated that anti-CTLA-4 antibodies in pathogen-free and germfree mouse models were less active when administered in combination with ATBs, because reduced the activation of splenic effector CD4+ T cells, and TILs (12). From this first preclinical evidence, the hypothesis emerged that the ATBs-related dysbiosis might reduce the diversity of gut microbiota thereby eliminating the most immunogenic bacteria (13).

Tinsley and colleagues analyzed 291 patients with advanced cancer treated with ICI (14). Patient ATB use was grouped into no ATB use, single course of ATB and cumulative ATB use, where ATBs were administered for $>7$ days or where patients received more than one ATB (either intravenous or oral). In the study, 92 patients (32\%) received antibiotics. Interestingly, patients who received a prolonged ATB treatment had the worst outcome (median OS 6.3 months, $\mathrm{P}=0.009$ ), although a single course of ATB did not. Thus, the protract ATB therapy may influence ICI efficacy due to the modifications in gut microbiota and, as a consequence, immune system activity. However, it could be also hypothesized that prolonged ATB treatment might be an epiphenomenon of an exhausted immune system more prone to infections. Therefore clinicians have to judiciously prescribe ATBs, considering also that human gut microbiota biodiversity may be reduced with modifications persisting up to 6 weeks (15).

Recently, various retrospective studies evaluated the impact of ATB use, in particular in patients affected by NSCLC treated with PD-1 inhibitors (16-18), as shown in Table 1. Most of these studies have analyzed the impact of early ATB use (before 1-2 months and 1 month after the start of immunotherapy) $(9,10,16,17,19)$, while Tinsley et al. focused on late exposure (from 2 weeks before until 6 weeks after ICI treatment initiation), highlighting the importance of a protracted analysis in order to monitor late effects (14). Collectively, these studies suggest that ATB use has a negative impact on outcomes in patients receiving ICI in terms of ORR $(10,13,20)$, PFS $(9,10,13,16,19-21)$, and OS $(9,13,16,17,19,21)$. In the study of Tinsley et al. (14), the majority $(40.2 \%)$ of treated infections originated from respiratory tract, but no other information were reported such as the microbiological diagnosis or the severity of the infection. In our opinion, if this data will be validated, it is crucial to individuate the correct approach to ATB therapy during ICI treatment: when is ATB use recommended? How long ATB treatment should last? Should immunotherapy be suspended during or after ATB use and in this case for how long?

Another fundamental issue not investigated extensively is the class of antibiotic prescribed. In fact, it is well recognized that various classes of ATBs have a role in the modulation of immune system. For example, macrolides prove to have a direct immunomodulatory activity, they inhibits the production of proinflammatory cytokines, transcription factors of inflammation such as nuclear factor kappa B (NF-kB) and infiltration of neutrophils from blood to tissue (22). Fluoroquinolones such as ciprofloxacin, moxifloxacin and levofloxacin have been demonstrated to dose-dependently inhibit the production of interleukin-1 (IL-1) and tumor necrosis factor (TNF)- $\alpha$ at therapeutic concentrations in monocytes and, at the same time, superinduce interleukin-2 (IL-2) in vitro, influencing the immune system (23). Doxycycline is an oral antibiotic of the tetracycline class, largely used for treatment of folliculitis induced by tyrosine-kinase inhibitors. In this case, folliculitis is not mediated by infectious agents and ATB effectiveness might be caused by anti-inflammatory activity (24). All these drugs might influence not only the gut microbiota but also the immune system, and, as a consequence, the tumor 
Table 1 Studies investigating the impact of antibiotic use during treatment with immune-checkpoint inhibitors

\begin{tabular}{|c|c|c|c|c|c|c|c|c|c|c|c|c|}
\hline Study & $\begin{array}{l}\text { Number } \\
\text { of patients } \\
\text { [NSCLC] }\end{array}$ & $\begin{array}{c}\text { Period of ATB } \\
\text { treatment }\end{array}$ & $\begin{array}{l}\text { Patients } \\
\text { treated with } \\
\text { ATB (\%) }\end{array}$ & \multicolumn{3}{|c|}{ ORR (\%) } & \multicolumn{3}{|c|}{ PFS (months) } & \multicolumn{3}{|c|}{ OS (months) } \\
\hline $\begin{array}{l}\text { Routy } \\
\text { et al. (9) }\end{array}$ & 249 [140] & $\begin{array}{c}\text { Within } 2 \text { months } \\
\text { before and } \\
1 \text { month after }\end{array}$ & $69(27.7)$ & NA & NA & NA & 4.1 & 3.5 & 0.017 & 20.6 & 11.5 & $<0.001$ \\
\hline $\begin{array}{l}\text { Tinsley } \\
\text { et al. (14) }\end{array}$ & 291 [64] & $\begin{array}{l}\text { Within } 2 \text { weeks } \\
\text { before and } \\
6 \text { weeks after }\end{array}$ & $92(31.6)$ & NA & NA & NA & 6.3 & 3.1 & 0.003 & 21.7 & 10.4 & 0.002 \\
\hline $\begin{array}{l}\text { Tinsley } \\
\text { et al. (19) }\end{array}$ & 305 [58] & $\begin{array}{c}\text { Within } 2 \text { weeks } \\
\text { before and } \\
6 \text { weeks after }\end{array}$ & $94(30.8)$ & NA & NA & NA & 5.8 & 3.2 & 0.049 & 21.4 & 10.4 & 0.001 \\
\hline $\begin{array}{l}\text { Zhao } \\
\text { et al. (20) }\end{array}$ & 109 [109] & $\begin{array}{l}\text { Within } 1 \text { month } \\
\text { before and } \\
1 \text { month after }\end{array}$ & 20 (18.3) & 22.5 & 15 & 0.092 & 9.6 & 3.7 & $<0.0001$ & 21.9 & 6.1 & 0.002 \\
\hline $\begin{array}{l}\text { Ouaknine } \\
\text { et al. (17) }\end{array}$ & 72 [72] & $\begin{array}{c}\text { Within } 2 \text { months } \\
\text { before and } \\
1 \text { month after }\end{array}$ & 28 (38.9) & NA & NA & 0.276 & 3.3 & 2.8 & 0.249 & 13.4 & 5.1 & 0.027 \\
\hline $\begin{array}{l}\text { Galli } \\
\text { et al. (18) }\end{array}$ & 157 [157] & $\begin{array}{l}\text { Within } 1 \text { month } \\
\text { before and } \\
3 \text { months after }\end{array}$ & $46(29.3)$ & 11.1 & 24.6 & 0.202 & 3.3 & 2.2 & 0.177 & 5.9 & 11.9 & 0.249 \\
\hline
\end{tabular}

ATB, antibiotics; NR, not reached; NA, not available; NSCLC, non-small cell lung cancer; ORR, overall response rate; OS, overall survival; PFS, progression free survival; RCC, renal cell carcinoma.

microenvironment.

For the future, prospective studies are needed to better understand the cross-talk between concomitant medications and comorbidities, tumor microenvironment and the gut microbiome, that might affect ICI activity. This is particularly important for studies in real-world practice, where a less selected group of patients is included with a higher risk of use of ATB (25). In this context, optimal ATB window, differences in ATB classes, route of administration, duration of ATB therapy need to be better defined. Prospective studies should also include examination of the composition of gut microbiota, that is emerging as a potential adjuvant treatment to ICI therapy. Meanwhile, in the absence of clear evidence, concomitant drugs, especially for a prolonged period or for repeated courses, during immunotherapy should be carefully evaluated. However, the use of ATB still remains strongly recommended in cases of symptomatic bacterial infections, as well as opportunistic infectious diseases that may develop in cases of immune depression, as observed in patients requiring prolonged therapies for severe immune-related adverse events.

\section{Acknowledgments}

None.

\section{Footnote}

Conflicts of Interest: The authors have no conflicts of interest to declare.

Ethical Statement: The authors are accountable for all aspects of the work in ensuring that questions related to the accuracy or integrity of any part of the work are 
appropriately investigated and resolved.

\section{References}

1. Henrique Corrêa L, Simões Heyn G, Grace Magalhaes K. The Impact of the Adipose Organ Plasticity on Inflammation and Cancer Progression. Cells 2019;8:662.

2. McQuade JL, Daniel CR, Hess KR, et al. Association of body-mass index and outcomes in patients with metastatic melanoma treated with targeted therapy, immunotherapy, or chemotherapy: a retrospective, multicohort analysis. Lancet Oncol 2018;19:310-22.

3. De Giorgi U, Procopio G, Giannarelli D, et al. Association of Systemic Inflammation Index and Body Mass Index with Survival in Patients with Renal Cell Cancer Treated with Nivolumab. Clin Cancer Res 2019;25:3839-46.

4. Farolfi A, Petrone M, Scarpi E, et al. Inflammatory Indexes as Prognostic and Predictive Factors in Ovarian Cancer Treated with Chemotherapy Alone or Together with Bevacizumab. A Multicenter, Retrospective Analysis by the MITO Group (MITO 24). Target Oncol 2018;13:469-79.

5. Rossi L, Santoni M, Crabb SJ, et al. High neutrophil-tolymphocyte ratio persistent during first-line chemotherapy predicts poor clinical outcome in patients with advanced urothelial cancer. Ann Surg Oncol 2015;22:1377-84.

6. Lolli C, Caffo O, Scarpi E, et al. Systemic ImmuneInflammation Index Predicts the Clinical Outcome in Patients with mCRPC Treated with Abiraterone. Front Pharmacol 2016;7:376.

7. Lolli C, Basso U, Derosa L, et al. Systemic immuneinflammation index predicts the clinical outcome in patients with metastatic renal cell cancer treated with sunitinib. Oncotarget 2016;7:54564-71.

8. Bigot F, Castanon E, Baldini C, et al. Prospective validation of a prognostic score for patients in immunotherapy phase I trials: The Gustave Roussy Immune Score (GRIm-Score). Eur J Cancer 2017;84:212-8.

9. Routy B, Le Chatelier E, Derosa L, et al. Gut microbiome influences efficacy of PD-1-based immunotherapy against epithelial tumors. Science 2018;359:91-7.

10. Elkrief A, Derosa L, Kroemer G, et al. The negative impact of antibiotics on outcomes in cancer patients treated with immunotherapy: a new independent prognostic factor? Ann Oncol 2019. [Epub ahead of print].

11. Wang Y, Wiesnoski DH, Helmink BA, et al. Fecal microbiota transplantation for refractory immune checkpoint inhibitor-associated colitis. Nat Med 2018;24:1804-8.

12. Vétizou M, Pitt JM, Daillère R, et al. Anticancer immunotherapy by CTLA-4 blockade relies on the gut microbiota. Science 2015;350:1079-84.

13. Derosa L, Hellmann MD, Spaziano M, et al. Negative association of antibiotics on clinical activity of immune checkpoint inhibitors in patients with advanced renal cell and non-small-cell lung cancer. Ann Oncol 2018;29:1437-44.

14. Tinsley N, Zhou C, Tan G, et al. Cumulative Antibiotic Use Significantly Decreases Efficacy of Checkpoint Inhibitors in Patients with Advanced Cancer. Oncologist 2019 Jul 10. [Epub ahead of print].

15. Modi SR, Collins JJ, Relman DA. Antibiotics and the gut microbiota. Journal of Clinical Investigation 2014;124:4212-8.

16. Hakozaki T, Okuma Y, Omori M, et al. Impact of prior antibiotic use on the efficacy of nivolumab for non-small cell lung cancer. Oncol Lett 2019;17:2946-52.

17. Ouaknine J, Helly De Tauriers P, Dumenil C, et al. MA10.03 Plasmatic Evaluation of the Intestinal Barrier and Blood Microbiota, and Antibiotic Use in Non-Small Cell Lung Cancer Patients Treated with Nivolumab. J Thorac Oncol 2018;13:S389-90.

18. Galli G, Triulzi T, Proto C, et al. Association between antibiotic-immunotherapy exposure ratio and outcome in metastatic non small cell lung cancer. Lung Cancer 2019;132:72-8.

19. Zhao S, Gao G, Li W, et al. Antibiotics are associated with attenuated efficacy of anti-PD-1/PD-L1 therapies in Chinese patients with advanced non-small cell lung cancer. Lung Cancer 2019;130:10-7.

20. Lalani AA, Xie W, Martini DJ, et al. Change in Neutrophil-to-lymphocyte ratio (NLR) in response to immune checkpoint blockade for metastatic renal cell carcinoma. J Immunother Cancer 2018;6:5.

21. Tinsley N, Zhou C, Villa S, et al. Cumulative antibiotic use and efficacy of immune checkpoint inhibitors in patients with advanced cancer. J Clin Oncol 2018;36:3010.

22. Giamarellos-Bourboulis EJ. Macrolides beyond the conventional antimicrobials: a class of potent immunomodulators. Int J Antimicrob Agents 2008;31:12-20.

23. Dalhoff A, Shalit I. Immunomodulatory effects of quinolones. Lancet Infect Dis 2003;3:359-71.

24. Robert C, Soria JC, Spatz A, et al. Cutaneous side-effects 
of kinase inhibitors and blocking antibodies. Lancet Oncol 2005;6:491-500.

25. De Giorgi U, Cartenì G, Giannarelli D, et al. Safety and

Cite this article as: Casadei C, Lolli C, Farolfi A. Immunecheckpoint inhibitors and the importance of concomitant medications: focus on antibiotics. Ann Transl Med 2019;7(Suppl 8):S339. doi: 10.21037/atm.2019.09.99 efficacy of nivolumab for metastatic renal cell carcinoma: real-world results from an expanded access programme. BJU Int 2019;123:98-105. 